\title{
L'objet ethnographique entre marché et patrimoine : cartographie de quelques enjeux
}

The ethnographical object between the market and heritage: a map and what is at stake

\section{Audrey Doyen}

\section{(2) OpenEdition}

\section{Journals}

Édition électronique

URL : http://journals.openedition.org/iss/590

DOI : $10.4000 /$ iss. 590

ISSN : 2306-4161

\section{Éditeur}

ICOM - International Council of Museums

Édition imprimée

Date de publication : 1 juin 2015

Pagination : 95-109

ISSN : 2309-1290

Référence électronique

Audrey Doyen, «L'objet ethnographique entre marché et patrimoine : cartographie de quelques enjeux », ICOFOM Study Series [En ligne], 43a | 2015, mis en ligne le 06 février 2018, consulté le 20 avril 2019. URL : http://journals.openedition.org/iss/590; DOI : 10.4000/iss.590 


\title{
L'objet ethnographique entre marché et patrimoine : cartographie de quelques enjeux
}

\author{
Audrey Doyen \\ Université de Neuchâtel - Suisse / Université Sorbonne \\ Nouvelle - France
}

\section{« Un musée qui n'achète pas est un musée mort »}

Ces mots prononcés par Pierre Rosenberg, directeur du Louvre, témoignent avec force de l'importance du lien entre les marchés d'art et les musées. Les rapports directs sont souvent niés et amoindris, notamment du côté de l'institution muséale, car comme l'expliquent André Gob et Noémie Drouguet (2010, p. 174) :

Le marché de l'art et des antiquités est avant tout un instrument financier; il n'a que peu à voir avec l'art ou le patrimoine.

La relation avec les marchés de l'art est même parfois crainte par les musées, qui y voient un risque accru d'être associés au trafic illicite de biens culturels. Pourtant, on peut observer comme le rappelle François Mairesse (2010, p. 44) :

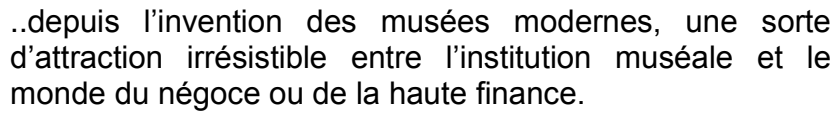

Cette relation se retrouve, dans le cas qui m'intéresse, entre les marchands d'art et les musées d'ethnographie qui exposent parfois les mêmes objets, attirent parfois les mêmes visiteurs, et échangent leurs idées sur les objets qui les intéressent. On pense bien évidemment à l'influence décisive qu'a eue le marchand d'art Jacques Kerchache dans le projet de l'actuel musée du quai Branly. Si c'est dans ce contexte passablement médiatisé que l'implication des marchands d'art dans l'institution du musée d'ethnographie s'est révélée, les contacts entre ces deux intermédiaires de l'objet non occidental datent de bien plus longtemps (Corbey, 2000 ; Penny, 2010). Leurs relations sont anciennes, comme le montrent par exemple les archives des musées d'ethnographie de Genève et Neuchâtel : dès la fondation de ces musées (respectivement 1901 et 1904), les directeurs s'emploient à créer des réseaux de contacts marchands plus ou moins en vue, de Jeanne Walschot à Bruxelles à Charles Ratton à Paris (Doyen, 2012).

Ces réseaux ne se sont pas éteints aujourd'hui. Musées et marchés sont toujours deux acteurs importants du champ de l'art non occidental, impliqués dans la production de la valeur monétaire, sociale et culturelle de l'objet. Ils sont tous deux des intermédiaires ou médiateurs (Heinich, 1997) - de l'objet extra-européen et se situent entre la création de l'objet et sa consommation par les collectionneurs, critiques ou visiteurs de musées. Quelles relations entretiennent-ils aujourd'hui ? Plus concrètement, quelles sont les modalités d'influences réciproques entre ces deux acteurs et leurs conséquences pour les musées et pour les objets concernés ? Ce sont ces questions qu'explore ma recherche en cours, dont les premières hypothèses sont exposées ici. Deux exemples nous 
permettent d'entrer dans certains cas de collaborations entre institutions muséales et milieux marchands: l'exposition Dogon présentée au musée du quai Branly en 2011 et l'exposition conçue par le Musée royal de l'Afrique centrale de Tervuren pour la Brussel Antiques and Fine Art fair de janvier 2014. Pour comprendre ces exemples, nous définirons le cadre général de la relation entre marchés et musées et les spécificités de chacun de ces deux acteurs. Cela nous permettra finalement de proposer quelques premiers éléments de compréhension tant des objectifs visés par les marchés et les musées que des stratégies mises en place pour les atteindre.

\section{Des galeristes dans les musées, des musées dans les foires}

Le premier cas qui nous intéresse est l'exposition Dogon proposée par le musée du quai Branly entre le 5 avril et le 24 juillet 2011. Cette exposition fut réalisée sous le commissariat d'Hélène Leloup, marchande et galeriste reconnue par ses pairs, qui mène des recherches sur les Dogons depuis de nombreuses années. Hélène Leloup avait déjà publié en 1994, soit dix-sept ans avant l'exposition au quai Branly, un ouvrage intitulé Statuaire Dogon. L'ouvrage en question est préfacé par William Rubin (directeur émérite du Musée d'art moderne de New York) et propose une contribution de Richard Serra (sculpteur) et de Georges Baselitz (peintre et collectionneur). La première partie de l'ouvrage est composée d'articles explicatifs de la culture dogon: histoire de la statuaire dogon et histoire de la découverte de l'Afrique, organisation sociale et religieuse des Dogons, fabrication des sculptures, artistes, esthétique, etc. le tout illustré de cartes des styles artistiques africains et dogons. La deuxième partie de l'ouvrage présente une centaine d'objets dogons, classés selon leurs styles et agrémentés de photographies pleine page. L'objectif de l'ouvrage publié en 1994 et celui de l'exposition et de son catalogue en 2011 sont clairement différenciés : dans le premier, Hélène Leloup se focalise sur la reconnaissance de l'art dogon en mobilisant des arguments divers - comme par exemple les similitudes entre arts occidental et dogon - afin de faire comprendre au lecteur l'importance de cet art. En 2011, il ne s'agit plus de faire reconnaître l'art dogon, mais d'apporter une seconde lecture. Celle-ci replace les objets dans leur contexte historique, afin de pointer la disparition inexorable de la culture dogon et le manque d'intérêt des chercheurs pour certaines parties de l'Afrique (2011, p. 13).

L'exposition Dogon au musée du quai Branly était construite en quatre parties. L'introduction retraçait l'histoire et les origines des Dogons. La deuxième partie présentait 133 sculptures classées selon des styles ou des aires géographiques spécifiques (Djennenké, Niongom, Tombo, Tellem, etc.). "L'imaginaire anthropologique », titre du troisième espace, était présenté par l'intermédiaire de deux ouvrages anthropologiques phares sur les Dogons, Le plateau central nigérien de Louis Desplagnes (1907) et Masques dogons de Marcel Griaule (1938). Finalement, l'exposition se concluait sur 140 objets illustrant non seulement la fascination des collectionneurs pour cet art, mais aussi la présence du mythe des origines dans chaque objet. L'exposition suscita l'engouement du public, puisque plus de 195000 visiteurs s'y rendirent en trois mois.

Le deuxième exemple sur lequel nous aimerions nous appuyer est celui de l'exposition présentée par le Musée royal de l'Afrique 
centrale de Tervuren à la Brussels Antiques and Fine Art fair (ciaprès BRAFA), du 25 janvier au $1^{\text {er }}$ février 2014. La BRAFA, qui a fêté sa $60^{\mathrm{e}}$ édition en 2015 , est une foire internationale d'art et d'antiquités qui se tient tous les ans à Bruxelles, où " toutes les œuvres d'art présentées sont à vendre " et qui offre "une très large variété de spécialités depuis l'Antiquité jusqu'au $\mathrm{XXI}{ }^{\mathrm{e}} \mathrm{s}$. » (BRAFAorganisation, 2015). L'objectif annoncé de l'édition de 2014 était de dépasser le seuil des 50000 visiteurs. Le musée fut l'invité d'honneur de la foire et présenta une exposition en quatre temps, dans une salle de l'espace Tour et Taxis. Accueilli par la girafe empaillée présentée autrefois dans l'exposition permanente du Musée royal de l'Afrique centrale, le visiteur se retrouvait face à quatre grandes vitrines et quelques éléments isolés, telle la maquette du projet de rénovation du musée. Les vitrines principales réunissaient parfois des ensembles d'objets a priori disparates et comportaient comme seule mention de texte un titre apposé sur le haut de la vitrine. Les objets n'étaient pas, ou peu, légendés et il fallait se rapporter aux fascicules posés sur une table de l'espace pour connaître les détails des pièces ainsi que la cohérence de leur association. Les concepteurs de l'exposition justifient cette absence par la volonté de garder des vitrines claires et lisibles que des cartels en trois langues (français, anglais et néerlandais) auraient alourdies. L'article paru dans le catalogue BRAFA 2014 précise que la présence du musée de Tervuren à la foire est l'occasion pour le visiteur de pouvoir observer quelques pièces du musée malgré la fermeture du bâtiment durant sa rénovation (BRAFA, 2014, p. 5). Ainsi, l'exposition présente quatre grandes thématiques chères au musée et définies par l'équipe de Tervuren : les pièces maîtresses des collections, considérées comme représentatives d'un certain imaginaire sur l'Afrique, les objets remarquables (par leur forme, leur taille, leur matériau) et donc souvent peu présents dans les collections ethnographiques, l'histoire des collections et, finalement, les instruments de musique, vaste ensemble des collections du Musée royal de l'Afrique centrale. L'ensemble se conclut sur la maquette du futur musée. La collaboration fut positive et l'exposition bien reçue puisqu'elle comptabilisa 30000 entrées pour une semaine de présentation.

\section{Caractéristiques des acteurs en jeu}

Ces deux exemples mettent en jeu deux types d'acteurs : les musées d'ethnographie et les marchands d'art, même si la situation ne peut se résumer à une simple dichotomie ${ }^{67}$. En outre, ils prennent place dans des lieux spécifiques (musées, foires d'art) dont l'emplacement géographique (Paris et Bruxelles) n'est pas anodin. Paris et Bruxelles sont deux lieux où se sont implantés de façon parallèle d'importants marchés de l'art non occidental et des musées d'ethnographie nationaux ou reconnus internationalement. II convient de s'attarder quelque peu sur quelques caractéristiques de ces acteurs, bien que certaines puissent sembler évidentes, pour comprendre les premiers enjeux de la relation entre les musées d'ethnographie et les marchands d'art non européen.

\section{Les musées : une relation forte à l'Etat}

La création et le développement des musées d'ethnographie bénéficient d'un modèle théorique qui trouve ses caractéristiques

\footnotetext{
${ }^{67}$ On trouve bien entendu tout un panel d'acteurs entre les musées et les marchands, telles les fondations privées souvent assimilées à l'institution muséale.
} 
dans la méthode de classement, I'histoire des collections, la politique de médiation, d'exposition et d'acquisition, etc. Cependant, il existe aussi un domaine important - la relation entre l'Etat et les institutions muséales - dans lequel les musées d'ethnographie européens, et notamment les musées d'ethnographie français, se distinguent. Nelia Dias (1989), pour exemple, fait remarquer en évoquant l'histoire du musée d'ethnographie du Trocadéro :

\begin{abstract}
La dichotomie collections publiques/collections privées a joué un rôle non négligeable en France et confère d'une certaine façon une spécificité au mouvement muséologique dans ce pays. En fait, notre analyse des collections anthropologiques et ethnographiques en France nous a permis de mettre en évidence l'emprise des autorités publiques sur les musées en général et les collections en particulier (Dias, 1989).
\end{abstract}

Cette remarque est reprise par Maureen Murphy (2009), qui oppose le modèle américain et son ouverture au privé au modèle français au fort centralisme étatique, sur la base des considérations de Pomian (1987, p. 301). On peut aujourd'hui discuter cette opposition peu nuancée, puisque depuis les années quatre-vingts les musées français connaissent des bouleversements dans la relation qui les lie à l'Etat ; Raymonde Moulin (1995) met en avant le caractère mixte, plutôt que totalement étatique, du fonctionnement du musée. En effet, dès les années quatre-vingts, des techniques anglo-saxonnes d'administration culturelle sont intégrées au musée (recours aux fonds privés, fondations privées, mécénat, etc.) et certains modèles de fonctionnement du musée sont calqués sur ceux du marché (audit, rentabilité, etc.) (Mairesse, 2010, p. 103-104). Ces transformations sont l'indicateur d'un rééquilibrage toujours en cours entre les sphères privée et publique. Ce rééquilibrage nous permet de comprendre l'historique et les enjeux actuels de la position - entre secteur public et privé - des musées impliqués dans nos exemples.

\title{
Marchands : la galerie, un acteur central et dynamique
}

Paris et Bruxelles sont non seulement des lieux où ont été fondés d'importants musées d'ethnographie, mais aussi des centres d'échange et de vente des objets ethnographiques dès leur arrivée en Europe. Aujourd'hui encore, ces deux villes ont conservé cette place centrale dans les ventes d'objets, comme le précise Maureen Zarember (2012) directrice de la Tambaran Gallery à New York au sujet de Paris :

\footnotetext{
Paris est une plate-forme importante pour l'art premier; chaque année les tendances s'en dégagent. Le marché français qui est composé de connaisseurs est plus traditionnel et établi que le marché new-yorkais, qui a vu une explosion des collectionneurs venant du monde de l'art moderne et contemporain (Zarember, 2012).
}

Le nombre important de galeries et d'événements ainsi que la présence marquée des départements art africain et art océanien de Sotheby's et Christie's illustrent ce propos. La plupart des plus grands marchands d'art extra-occidental (en termes de chiffre d'affaires et de reconnaissance internationale) se trouvent concentrés à Paris et Bruxelles et ils entretiennent une concurrence qui s'est développée dès le $19^{\mathrm{e}}$ siècle entre les marchands de ces deux villes. 
Il convient de s'attarder brièvement sur le terme de marchand : celuici regroupe un certain nombre de définitions et il est intéressant dans cette analyse de circonscrire la catégorie. Cette limitation peut s'opérer sur la base de trois axes: les définitions législatives et académiques qui définissent le marchand, le type de marchand auquel nous nous intéressons et finalement les définitions tenues par les acteurs et leurs positions sur leur profession et son objet.

Tout d'abord, il existe certaines législations qui définissent, de façon plutôt générale, la profession de marchand d'art extra-occidental en l'associant généralement à celle d'antiquaire. Au niveau académique, la loi française fait référence dans les milieux francophones - c'est notamment celle que l'on trouve chez Sophie Cazaumayou (2009, p. 44) dans son étude sur le marché de l'art océanien: selon cette définition, le marchand serait «toute personne dont l'activité professionnelle comporte la vente ou l'échange d'objets mobiliers usagés ou acquis de personnes autres que celles qui les fabriquent ou en font commerce " (Code pénal français, article 321-7). Cette définition juridique, volontairement englobante dans le cas des dispositions sur le recel, ne peut être appliquée ainsi à notre analyse. II convient de préciser cette définition juridique ; le marchand, selon notre analyse, est un individu qui non seulement propose des objets à la vente, mais en fait commerce et donc en tire une activité lucrative. Celle-ci est sa source principale de revenus, ce qui nous permet d'exclure de la catégorie les particuliers s'adonnant à un passe-temps occasionnel.

Deux types de marchands sont compris dans cette définition : le galeriste et le commissaire-priseur, tous deux rattachés à des lieux, la galerie et la maison de ventes aux enchères. Si les maisons de ventes aux enchères n'apparaissent pas dans les deux exemples développés ici, il est intéressant de relever quelques points caractéristiques de cette institution, qui sont importants pour comprendre le paysage général du marché de l'art non occidental, ainsi que la position de la galerie dans ce paysage. Davantage réglementée que celle de marchand, la profession de commissairepriseur s'organise en une Chambre Nationale, organisation faîtière reconnue et structurée qui assure la transparence d'un grand nombre de points (les marges, par exemple, sont connues de tous). La profession est en outre caractérisée par un esprit de corps très marqué (Quemin, 1997) ; lors d'une vente, le commissaire-priseur représente sa profession : il ne peut vendre n'importe quel objet sous peine de la décrédibiliser, contrairement au marchand qui n'engage que sa personne et son propre commerce. A cause de cet aspect plus " officiel ", les ventes aux enchères sont peu à peu devenues des représentations des tendances générales, ce qui a fait dire à Beurdeley (1988, p. 16) que « c'est l'hôtel des ventes qui permet au public de prendre la température d'une tendance à une époque donnée ».

L'institution qu'est la maison de ventes aux enchères nous permet de comprendre certains enjeux de la relation entre musées d'ethnographie et marchés d'art non occidental ; en effet, dans le paysage du champ de l'art non occidental, relativement récent et donc moins organisé et moins reconnu que celui de l'art classé ${ }^{68}$, les galeries se retrouvent entre deux autres médiateurs, soit fortement

\footnotetext{
${ }^{68}$ Selon Raymonde Moulin (1997, p. 15), ce terme englobe les peintures et sculptures anciennes ainsi que l'art moderne.
} 
institutionnalisés (la maison de ventes aux enchères), soit liés à l'Etat (le musée). Si cette situation peut désavantager les galeristes, elle les place aussi dans la position du seul acteur indépendant (de l'Etat ou d'un employeur), les dotant de certains avantages: liberté et rapidité d'action et de choix, allègement de la charge administrative inhérente à toute institution, etc. En outre, cette situation peut aussi, de façon paradoxale, fournir des modèles de stratégies à adopter pour faire gagner à la galerie la crédibilité que son statut non institutionnel lui retire, comme nous le verrons plus loin.

Finalement, le domaine de l'art extra-occidental souffre d'un problème lexical important qui reflète les problèmes de catégorisation de ce type d'objets ${ }^{69}$. Le lexique usité par chaque acteur est représentatif du contexte de son emploi ou des représentations que le locuteur se fait de l'objet (voire des sociétés productrices de cet objet). Les marchands définissent leur profession par le type d'objets vendus, par la définition des sociétés productrices de l'objet ou, plus rarement, par le public visé : ainsi, presque inexistants sont les marchands qui parlent « d'objets ethnographiques ». Ceux-ci privilégient, en français, le terme d'art extra-occidental avec la spécification de la provenance des objets vendus (la provenance étant un critère de valeur commerciale de l'objet): Didier Claes parle d'art africain, Sarah de Monbrison d'african, oceanic and north american art, etc. Art primitif et art premier sont des termes couramment utilisés en français : art primitif chez Lucas Ratton ou Schoffel-de Fabry, par exemple, ou arts premiers chez Yann Ferrandin (BRAFA, 2014). Le terme d'art tribal ne s'entend que rarement chez les francophones. En revanche, il s'est davantage développé dans les milieux anglo-saxons sous l'influence des thèses de l'anthropologie sociale britannique. Le terme est aujourd'hui couramment usité dans les textes ou discours anglais : ainsi dans le catalogue en anglais de la BRAFA 2014, la plupart des galeristes portent leur intérêt sur the art of ancient tribal societies ou se définissent comme tribal art dealer (BRAFA, 2014, pp. 190, 214, 448, 456). Alors que l'ancienneté des pièces est reconnue comme un élément important de la valeur des objets, (Cazaumayou, 2009 ; Derlon \& Jeudy-Ballini, 2008), ce critère est complètement passé sous silence dans la définition générale de l'objet par la plupart des marchands. Bernard Dulon est un des seuls galeristes à mentionner l'ancienneté comme critère de définition des objets vendus, alors que les autres galeristes entretiennent un flou sur la question, évitant de déterminer l'ancienneté ou les périodes historiques pertinentes d'un objet d'art non occidental. C'est que l'ancienneté définit, bien que très vaguement, une temporalité, alors que, comme le relevaient Brigitte Derlon et Monique Jeudy-Ballini (2008, pp. 87-89), le « récepteur » d'un objet non occidental (qu'il soit visiteur, collectionneur, observateur, possesseur, etc.) mobilise un imaginaire qu'il projette sur l'objet. Le rêve et le mystère sont au centre de la fascination pour l'art non occidental et la définition trop précise de certains paramètres nuit à l'intérêt porté à cet art. En outre, le flou entretenu autour de la définition des objets vendus laisse au marchand une marge de manœuvre qui lui permet de se repositionner rapidement sur le marché en cas de modifications des intérêts, comme le développement observé récemment d'une

\footnotetext{
${ }^{69}$ Voir par exemple Sally Price (1995) qui expose l'usage des différentes terminologies dans le milieu, sans les attribuer spécifiquement à l'un ou l'autre acteur en particulier. Dans la sphère académique, le problème est prégnant comme le discute Adriaan Claerhout (1965, p. 438) qui finit par qualifier primitif - et tous les termes s'y rapportant - de that damned word.
} 
attention accrue pour l'art non occidental plus contemporain (Parcours des mondes, 2013).

\section{Cartographie d'un jeu à double sens}

\section{Crédibilité et reconnaissance}

Nombreux sont les galeristes à critiquer l'institution muséale et son immobilisme, son académisme ampoulé et empesé, comme par exemple Hélène Leloup dans sa publication de 1994. Cependant, nombreux aussi sont les marchands à reconnaître l'exposition de pièces dans des musées, l'expertise dans un catalogue ou, mieux, la responsabilité d'une exposition, comme autant de signes d'une reconnaissance et d'une validation de leur travail, comme nous le confiaient la plupart des galeristes en disant « nous on a intérêt [à vendre des objets à des musées], parce que ça fait prestigieux $"{ }^{70}$ ou :

On ne peut pas nier que d'un point de vue du prestige, de voir sa pièce dans un musée, c'est assez fou. II y a quelque chose quand même!

Un galeriste reconnu nous affirmait récemment qu'avoir une salle à son nom dans un musée, par le legs d'une collection personnelle par exemple, ou avoir certains de ses objets exposés dans un musée est considéré comme la réussite ultime à laquelle peut aspirer un marchand. Qu'est-ce donc que "ce quelque-chose quand même " et qu'est-ce que l'institution muséale apporte aux galeristes ?

La galerie ne possède pas, contrairement à la maison de ventes aux enchères, des caractéristiques institutionnelles traditionnelles et historiquement ancrées. Elle ne possède pas non plus le pouvoir du musée de consacrer des œuvres en tant qu'art ou patrimoine. Si dans le cas des arts non occidentaux les marchands sont souvent considérés comme les moteurs de la reconnaissance de cet art (Fossey, 2011), c'est la création d'un musée qui est venue consacrer la pertinence de leur travail, une lacune que les galeristes ont remarquée et qu'ils tentent, depuis quelques années, de combler en instaurant diverses stratégies qui permettent d'apporter la crédibilité que le caractère non institutionnel de la profession leur retire. Ce désir s'est aussi développé en réaction à certains scandales plus ou moins récents d'arnaques ainsi que dans le but de se différencier des "bananas », ces marchands ambulants sans attache (c'est-àdire sans galerie) régulièrement accusés de vendre des faux, trop chers, sans informations, décrédibilisant ainsi tout le champ de l'art extra-occidental. Plusieurs points ont été développés - et sont toujours en cours de développement - sur le modèle d'institutions déjà existantes, tels les musées, et sur le modèle d'autres champs, l'art classé et l'art contemporain notamment. Cette stratégie se concentre sur plusieurs éléments dont les principaux sont la provenance des pièces, la lutte contre les faux et une meilleure définition de la profession.

Afin d'augmenter la transparence au sujet de la provenance des pièces et contrairement à ce qui était la tendance il y a quelques décennies, celle-ci est de plus en plus régulièrement citée comme c'est le cas dans les expositions ou catalogues de musées. Cependant, cette tendance tend parfois à l'absurde du côté des

\footnotetext{
${ }^{70}$ Toutes les citations sont extraites d'entretiens formels et informels menés avec des galeristes parisiens et bruxellois entre 2013 et 2014 .
} 
marchands, quand par exemple la provenance citée est celle d'une " collection privée " ou celle... d'un autre marchand. En effet, les reproches faits généralement aux marchands quant à la provenance des objets portent sur l'histoire de l'objet avant son arrivée en Occident (vol, spoliation, etc.). Ces reproches sont adressés par le monde scientifique, mais aussi par les collectionneurs de plus en plus demandeurs de certaines informations. Ainsi un galeriste confirme la hausse de cette tendance et les abus qui peuvent en découler :

Beaucoup de collectionneurs veulent des provenances avérées et parfois ils se retrouvent avec des fausses provenances, parce que les gens en veulent, donc on leur en donne!

L'indication de la provenance peut aussi être détournée pour devenir le moyen de citer le pedigree d'une œuvre, c'est-à-dire la valeur ajoutée que l'objet acquiert après être passé en des mains (re)connues. Ces indications de provenance viennent enrichir des légendes de plus en plus fournies servant aussi à lutter contre les ventes de faux. Si le mode de présentation des objets n'a que peu changé - le dénuement est toujours plébiscité -, les catalogues des pièces abondent en effet de légendes où figurent généralement la liste exhaustive de la littérature sur le sujet ainsi que des expositions qui ont été consacrées, prouvant ainsi que l'objet a été reconnu par des spécialistes ou des institutions notables. Ainsi les expositions de pièces ou les catalogues publiés par les galeries deviennent de plus en plus référencés, car comme nous déclarait un galeriste :

L'idée c'est qu'on apprenne quelque chose. "Bois rouge, patine d'usage ", ça permet juste comme les catalogues de ventes de savoir, mais pas d'apprendre quelque chose. Avant, ce qui importait, c'était purement l'esthétisme; aujourd'hui les gens cherchent à savoir ; ils sont parfois hyper-pointus.

Finalement, afin de garantir la qualité des pièces, les objets présentés dans les foires sont soumis à un strict processus d'évaluation par des comités - généralement composés d'experts ou de conservateurs de musées - que ce soit lors des foires d'art préexistantes comme BRAFA, TEFAF ou dans les cas où les marchands créent ou développent des foires centrées sur l'art non occidental, comme Bruneaf (Brussels non European art fair) à Bruxelles. Ainsi, un des premiers actes de Didier Claes, nouveau président de la foire Bruneaf, fut de créer un comité d'experts, comme il l'explique au Journal des Arts (Potard, 2014) :

\begin{abstract}
Dès janvier, pour le Winter Bruneaf, les experts vont circuler dans les galeries pour faire retirer les pièces fausses ou n'étant pas d'assez bonne qualité. Le comité d'experts passera la veille de l'ouverture officielle puis fera des visites surprises. Si un exposant replace une pièce retirée, il sera exclu de l'association. Le nerf de la guerre, dans ce genre d'évènement, c'est la lutte contre le faux.
\end{abstract}

Ainsi, les galeristes sont poussés - par leur situation entre deux acteurs fortement institutionnalisés - à élever leur niveau d'exigences ou, du moins, à imiter des fonctionnements propres à la sphère muséale, dont la crédibilité et le caractère institutionnel sont les garants d'une certaine scientificité. 
Un autre exemple de ces emprunts est le transfert de savoir-faire: celui-ci s'illustre dans la collaboration de plus en plus soutenue et de plus en plus régulière des marchands et des professionnels de musées, mais aussi dans le transfert de personnel d'un domaine à l'autre: il n'est pas rare que des collaborateurs de musées soient engagés en galerie ou que, comme dans le cas de l'exposition Dogon au musée du quai Branly, des marchands soient engagés pour des évènements ou expositions muséales. Le domaine de l'exposition est en effet particulièrement concerné par ce transfert de savoir-faire : les galeristes se calquent de plus en plus sur un mode de fonctionnement déjà utilisé par deux institutions reconnues : les musées et les maisons de ventes. Si la création d'un espace d'exposition pour la vente des pièces n'est pas un fait nouveau du côté des marchands (la galerie mettant en avant l'aspect visuel, tant l'esthétique de l'objet que la scénographie qui l'entoure, ce qui est non négligeable lors d'une vente), les galeristes conçoivent de plus en plus souvent l'exposition des pièces sur le modèle muséal avec les développements conséquents de légendes et cartels, mais aussi la mise en vitrine des objets, la documentation, une cohérence thématique, historique, etc. - de l'ensemble, tendant ainsi de plus en plus à de véritables expositions, titrées et accompagnées de catalogues. C'est ainsi ce que Serge Schoffel a mis en place lors de la BRAFA 2014 avec son exposition "Vodun », répondant à une promesse d'évolution faite à ses clients (BRAFA, 2014, p. 452) :

The gallery is fulfilling the promise to develop welldocumented thematic exhibitions and will unveil the mysterious side of Africa with "Vodun Fon: between art and matter".

Cette exposition de galeriste dans une foire d'art est une des premières à pousser la cohérence thématique de l'ensemble au point de présenter des objets non mis en vente mais destinés à documenter et compléter l'ensemble de l'exposition - se plaçant ainsi en porte-à-faux des déclarations de la foire selon lesquelles " tous les objets exposés sont à vendre " (BRAFA-organisation, 2015).

Finalement, un autre moyen mis en place par les galeristes pour gagner en crédibilité consiste à s'associer les institutions muséales, lors d'évènements, de ventes, d'expertises, etc. Cela ne signifie pas forcément l'exposition de pièces dans un grand musée, mais la mention régulière des coopérations ou l'invitation d'un musée tel que le Musée royal de l'Afrique centrale dans une foire telle que la BRAFA, de façon à répondre à des objectifs différents, par une collaboration commune.

Le musée, par son lien à l'Etat, son caractère non lucratif et donc désintéressé de tout aspect financier, homologue et hiérarchise les valeurs esthétiques (Moulin, 1997, p. 7). II consacre des artistes ou des champs artistiques et les légitime en tant qu'art ou patrimoine. Ainsi, en s'associant sa crédibilité, en adoptant son mode de fonctionnement ou ses savoir-faire - autant en matière de publications que d'expositions -, le galeriste peut, à travers des collaborations mais aussi une circulation d'idées et de personnes, atteindre une forme officielle de reconnaissance dans un paysage où peu d'éléments sont institutionnalisés.

\section{Spécialisations des connaissances et accès au public}


De l'autre côté, nombreux sont les musées à critiquer parfois vertement les marchands: arnaques des collectionneurs, mise en circulation de faux, vénalité, augmentation des prix, etc. Cependant, nombreux sont aussi les musées à solliciter les marchands pour l'expertise d'objets, l'écriture d'un article dans un catalogue ou le commissariat d'une exposition, comme dans le cas de l'exposition Dogon au musée du quai Branly. C'est que certains marchands mobilisent des connaissances extrêmement pointues dans des domaines très précis de l'art extra-occidental. L'exemple de ladite exposition est, à ce titre, emblématique : Hélène Leloup a développé tout au long de son activité de galeriste des connaissances pointues sur les Dogons, légitimant le choix de la nommer commissaire de l'exposition en 2011. La galeriste a ainsi repris les connaissances qu'elle avait accumulées ou les productions qu'elle avait déjà faites pour la conception de l'exposition et du catalogue de l'exposition. Le résultat est sans appel: l'exposition du musée du quai Branly présente les mêmes objets que ceux choisis par Hélène Leloup pour sa publication sur les Dogons en 1994. Seuls cinq objets présentés dans la publication n'ont pas été retenus pour l'exposition. Cette similitude se retrouve jusque dans le catalogue de l'exposition, qui adopte la même mise en page que la publication de 1994 : les sculptures présentées en pleine page en 1994 le sont à nouveau en 2011. Les articles de la publication Statuaire Dogon sont les mêmes que les articles proposés par Hélène Leloup dans le catalogue de l'exposition Dogon. Le processus observé chez les galeristes et qui consiste à reprendre certains éléments muséaux - connaissances, savoir-faire, productions - se retrouve dans cet exemple où, à l'inverse, les connaissances de la galeriste sur la thématique de l'exposition, mais aussi sa vision de leur présentation, sont reprises dans l'institution muséale.

L'apport des galeristes aux musées ne se situe pas uniquement dans leurs connaissances pointues sur certaines thématiques. Les marchands disposent aussi de davantage de marge de manœuvre pour l'expertise des pièces, comme le confirme Aurélien Gaborit dans un entretien donné à une chercheuse au sujet de l'expertise de pièces boli :

\begin{abstract}
Je dois dire que je suis plutôt content et en même temps un peu triste que le musée n'ait pas fait tout le travail qu'a fait Johann Lévy à l'époque sur les Boli, enfin je veux dire tout le travail de recherche, d'analyse scientifique sur un certain nombre de pièces qu'il a présentées donc dans son exposition sur les Boli. [...] Et oui, il a été plus vite, parce qu'ici c'est extrêmement long d'organiser tout ça, il faut un budget, un dossier, etc. (Fossey, 2011, p. 255)
\end{abstract}

Cette remarque démontre que l'expertise des marchands est légitime pour les professionnels de musée aujourd'hui ; les marchands sont reconnus comme plus dynamiques, plus rapides, et avec une possibilité d'innovation que l'administration muséale ne permet pas. Ainsi, ce sont les milieux marchands qui lancèrent les datations d'objets au carbone 14 et ce sont aujourd'hui toujours les milieux marchands qui effectuent la plupart des expertises de pièces en musée. Hélène Leloup (2011, p. 13) souligne dans son ouvrage que certains terrains sont peu explorés par les ethnologues et que ce sont les marchands qui les investissent, les récupèrent et les développent, devenant ainsi de véritables références dans leur domaine. 
La spécialisation, les connaissances et les marges de manœuvre que l'indépendance des marchands leur permet en font des partenaires de choix pour les musées lorsqu'il s'agit d'accéder à certains objets, que ce soit dans le domaine de l'acquisition ou dans celui des expositions. Ainsi certains marchands font partie des réseaux officiels (sociétés des amis, mécènes, etc.) ou non officiels des musées, comme Hélène Leloup, membre de la commission d'acquisition et du conseil d'administration de la Société des Amis du musée du quai Branly. Ils leur permettent d'accéder à certains objets inaccessibles autrement, comme le confirme Aurélien Gaborit :

En général, ils sont au courant en avance, ils nous disaient attention, cette année il y a un objet important qui va sortir, et là, on regardait et ça convenait ou pas (Fossey, 2011, p. 143).

Ce modèle est en effet largement adopté au Musée du quai Branly où la Société des Amis du musée a créé en 2008 le Cercle Claude Lévi-Strauss. Celui-ci comprend une vingtaine de membres sélectionnés dans les milieux d'antiquaires, de galeries ou de collections privées. Les membres du Cercle ont la possibilité de proposer des œuvres aux conservateurs qui selon, leurs besoins et désirs d'acquisition, peuvent soumettre la proposition au vote de l'ensemble de l'association. Le musée applique là un modèle proche du modèle américain où l'implication de la société civile est un aspect important de la gestion du musée.

Cette collaboration ne sert pas les seuls intérêts de l'acquisition, mais permet aussi l'accès aux objets dans le cas de prêts pour des expositions ou pour illustrer des catalogues. Les galeristes peuvent situer le " stock dormant » d'objets, c'est-à-dire la multitude d'objets répartis chez les collectionneurs ou chez eux-mêmes. De par leur profession, ils doivent rester à l'affût des pièces mises en vente, des collections qui se construisent, des goûts des collectionneurs, etc.

Finalement, la collaboration entre les musées et les galeristes leur apporte à tous deux l'accès à un public élargi. En effet, si les publics sont en grande partie les mêmes dans les deux cas (de nombreux collectionneurs attestent fréquenter aussi bien les musées que les galeries ou les foires), le public charrié par le musée est en général plus nombreux et plus diversifié. Ainsi, la collaboration d'un marchand avec un musée, dans le cas d'expositions, mais aussi d'évènements, accroît sa visibilité et élargit sa clientèle. Cet intérêt ne fonctionne pas à sens unique, comme nous le prouve la participation du Musée royal de l'Afrique centrale à la BRAFA: l'exposition visait la présentation générale du musée ainsi que le partage d'informations quant à sa rénovation. Cette participation relève entre autres d'une opération de communication visant à faire connaître le musée à un autre public (les amateurs d'art) à travers la présentation de l'histoire des collections du musée ainsi que de ses pièces maîtresses, mais aussi à faire part de sa fermeture et de sa rénovation, autant au public averti qu'au grand public, comme nous le confirmait dans un entretien Julien Volper, participant au projet :

Le projet tombait plutôt bien puisque le musée venait de fermer et c'était plus ou moins le premier événement important après cette fermeture. On touchait aussi un autre public avec BRAFA : c'est un public d'amateurs d'art.

La spécialisation des connaissances du marchand, son imbrication dans un réseau, sa faculté à réagir rapidement et par d'autres 
moyens que ceux dont dispose l'institution muséale en font un partenaire important pour les musées. En outre, l'accès à des publics différents par leur objectif (acheter ou s'informer), mais identiques par leur intérêt (l'art non occidental), sert leurs buts à tous deux, qu'ils soient d'informer, de diffuser ou d'attirer.

\section{Quelles conséquences pour les objets, quelles conséquences pour les musées?}

A travers ces premières observations et l'analyse préliminaire que nous en avons fait, nous pouvons pointer certains éléments importants de la collaboration entre les musées d'ethnographie et les marchands d'art non occidental: conséquences sur le système, sur l'objet, sur notre rapport à ce patrimoine souvent controversé, sur les aspects déontologiques et éthiques de la profession.

La méthode ethnographique utilisée pour ces analyses impose de prendre en compte les représentations, les pratiques et les discours, par un terrain comprenant des observations, des entretiens ou la participation et l'intégration aux différents milieux, autant muséaux que marchands. Cette analyse préliminaire de notre recherche de thèse en cours met en évidence, à travers les deux exemples largement développés de l'exposition Dogon et de la BRAFA, le fossé entre le discours des acteurs qui fustigent souvent l'autre partie et les pratiques de chacun, où l'on observe des collaborations soutenues et régulières, tant pour améliorer certaines productions (expositions, publications) que pour atteindre certains objectifs (documentation, communication, information).

La partie muséologique de ce travail interdisciplinaire impose quant à elle de prendre en compte ces observations ethnographiques pour en saisir les conséquences et les implications sur l'institution muséale. Ces conséquences, tant sur l'institution que sur l'objet, sont multiples. Tout d'abord, il s'agit de se souvenir que le modèle de fonctionnement du musée tel qu'il s'est développé dans les années quatre-vingts a non seulement permis, mais aussi encouragé, l'ouverture à ce genre de collaboration. Cependant, il s'agit de ne pas oublier que l'intérêt de cette collaboration n'est pas à sens unique, comme le rappelle le galeriste Yann Ferrandin dans la description de son activité (BRAFA, 2014, p. 214) :

His active policy of lending to major exhibitions at some of the most important American and European museums has earned him to the recognition of the prestigious institutions whose collections he regularly enriches.

Cette citation nous rappelle que si le galeriste permet l'enrichissement des collections de certains musées, c'est aussi un moyen pour lui d'arriver à une reconnaissance professionnelle. Le double mouvement et les intérêts multiples d'une collaboration entre marchés et musées sont contenus dans cette phrase.

Cette collaboration n'est pas sans conséquences sur notre façon d'appréhender l'objet au centre de l'attention du musée, du galeriste et du consommateur, qu'il soit collectionneur ou simple curieux. Cela nous rappelle que l'objet possède une valeur monétaire, liée à ses qualités intrinsèques ainsi qu'à la valeur de ses matériaux, qui est en fait peu importante pour la valeur marchande de l'objet. C'est que ces objets mobilisent une autre valeur, la valeur affective, liée au 
désir d'acquérir, à la représentativité de l'objet dans un ensemble, à son importance dans l'histoire humaine, à des modes, aux arguments mobilisés dans des contextes donnés, etc. L'institution muséale participe à la construction de cette valeur, en consacrant certains objets comme dignes d'être conservés, étudiés, montrés. Cependant, le marchand est aussi un acteur important de la constitution de cette valeur, car il participe à la visibilité et à la reconnaissance de certains objets par rapport à d'autres. Dans le cas de l'art non occidental, il est admis que ce sont les milieux marchands qui ont réussi à faire reconnaître ces objets comme dignes d'une attention non plus seulement portée sur les informations que les objets fournissent sur une culture, mais bien pour des critères intrinsèques, principalement esthétiques.

Une fois entré au musée, l'objet acquiert une nouvelle valeur : une valeur culturelle qui l'institue désormais comme partie du patrimoine. Dominique Poulot (2005) nous rappelle ce paradoxe: le musée consacre un objet qui y est entré « à la fois selon un projet propre et au hasard des conduites d'agents qui lui sont extérieurs ". Le marchand est l'un de ces critères aléatoires et c'est en quoi sa relation avec le musée est importante. Les milieux marchands ont créé et continuent de créer des arguments, des contextes et, au final, de la valeur, que le musée vient consacrer et, inversement, le musée offre aux milieux marchands des stratégies, des fonctionnements qui leur permettent de créer davantage d'arguments.

Si Krzysztof Pomian (1992) affirme que l'on ne peut saisir la spécificité du marché sans tenir compte du musée, nous voyons à travers ces premières analyses d'une recherche encore en cours que l'on ne peut pas non plus comprendre le fonctionnement de l'institution muséale sans tenir compte du marché. Ceci nous amène à envisager la définition de patrimoine comme mouvante et dynamique, dépendante d'acteurs et de réseaux externes aux musées, mais aussi à prendre conscience que la séparation entre argent et culture, entre sphère privée et sphère publique, entre aspects économiques et aspects culturels est impossible en l'état et que les relations, qu'il s'agisse de collaboration ou de concurrence, servent les objectifs et les intérêts des deux parties.

\section{Références}

Beurdeley, M. (1988). Trois siècles de ventes publiques. Fribourg: Office du livre.

BRAFA (Eds). (2014). BRAFA: Art fair 2014. Catalogue. Bruxelles: Delen Private Bank.

BRAFA - Organisation: Qui sommes-nous ?. (2015). BRAFA Art Fair. Retrieved from http://www.brafa.be/DesktopDefault.aspx?tabid=200

Cazaumayou, S. (2009). Objets d'Océanie: Regards sur le marché de l'art primitif en France. Paris: L'Harmattan.

Claerhout, A. (1965). "Discussion of a problem posed by Adriaan G. H. Claerhout: The Concept of Primitive Applied to Art. " Current Anthropology, 6, 4, 432-438.

Corbey, R. (2000). Tribal Art Traffic: A chronicle of Taste, Trade and Desire in colonial and post-colonial Time. Amsterdam: Royal Tropical Institute-The Netherlands.

Code pénal français. Version consolidée au 26 juin 2015. (2015). Livre III. Consulté à partir de http://www.legifrance.gouv.fr/affichCode.do ?cidTexte=LEGITEXT0000 06070719

Derlon, B., \& Jeudy-Ballini, M. (2008). La passion de l'art primitif: Enquête sur les collectionneurs. Paris: Gallimard. 
Dias, N. (1989). « Série de crânes et armées de squelettes: Les collections anthropologiques en France dans la seconde moitié du XIXe siècle. " Bulletins et mémoires de la société d'anthropologie de Paris, 1, 3-4, 203-230.

Doyen, A. (2012). Le musée d'ethnographie de Genève et ses marchands de 1901 à 1951: Politique d'acquisition et valeur de l'objet ethnographique. Université de Neuchâtel: travail de mémoire non publié.

Fossey, E. (2011). Paris XXle siècle, capitale du monde des arts d'Afrique Noire: Le rôle de la scène artistique parisienne dans la construction de la valeur des Arts d'Afrique Noire. Thèse effectuée sous la direction de Bruno Péquignot, Paris 3 -Sorbonne Nouvelle, non publiée.

Gob, A. \& Drouguet, N. (2010). La muséologie: Histoire, développements, enjeux actuels. Paris: Armand Colin.

Heinich, N. (1997). «Pourquoi la sociologie parle des œuvres d'art et comment elle pourrait en parler. "Sociologie de l'art, 10, 11-23.

Leloup, H. (1994). Statuaire dogon. Strasbourg: D. Amez.

Leloup, H. (2011). Dogon. Paris: Somogy/Musée du quai Branly.

Mairesse, F. (2010). Le musée hybride. Paris: la Documentation française.

Moulin, R. (1995). De la valeur de l'art : recueil d'articles. Paris: Flammarion.

Moulin, R. (1997). L'artiste, l'institution et le marché. Paris: Flammarion.

Murphy, M. (2009). De l'imaginaire au musée: Les arts d'Afrique à Paris et à New York (1931-2006). Dijon: les Presses du réel.

Parcours des mondes: Le salon international des arts premiers. Catalogue. (2013). Arquennes: Tribal art Management.

Penny, G. (2010). Objects of culture: Ethnology and ethnographic museums in imperial Germany, Chapel Hill: the University of North Carolina Press

Pomian, K. (1987). Collectionneurs, amateurs et curieux: Paris, Venise: XVlème-XVIIlème siècles. Paris: Gallimard.

Pomian, K. (1992). " L'art: Entre le marché et le musée. » Dans L. BertrandDorléac (Ed.). (1992). Le commerce de l'art de la Renaissance à nos jours (pp. 9-33). Besançon: Ed. de la Manufacture.

Potard, M. (2014, 17-30 janvier). "Entretien: Didier Claes, antiquaire à Bruxelles, président de Bruneaf, vice-président de Brafa. " Le Journal des arts, 32.

Poulot, D. (2005). « Les mutations de la sociabilité dans les musées français et les stratégies des conservateurs, 1960-1980. " Dans R. Moulin. (Eds.), Sociologie de l'art. Ministère de la Culture et de la Communication \& Société française de Sociologie (pp. 95-109). Paris: l'Harmattan.

Price, S. (1995). Arts primitifs; regards civilisés. Paris: Ecole nationale supérieure des Beaux-Arts.

Quemin, A. (1997). Les commissaires-priseurs: la mutation d'une profession. Paris: Anthropos.

Zarember, M. (2012, septembre). «Paris, nombril des mondes. » Beaux-Arts magazine, 134.

\section{Résumé}

Depuis quelques années, les remises en cause des musées d'ethnographie se multiplient; s'interroger sur leurs collections, c'est s'interroger sur notre manière d'appréhender ces objets : quelle valeur - culturelle, affective, monétaire, scientifique - leur attribue-t-on et pourquoi ? La recherche académique a déjà mis en avant certaines de ces valeurs et les réseaux complexes au sein desquels elles se constituent : collectionneurs, musées, marchands, publics, etc. Cet article présente les résultats intermédiaires d'une recherche en cours sur deux de ces acteurs: les musées d'ethnographie et les marchands d'art non occidental, ceci afin de comprendre les modalités de leur relation. Deux exemples de collaboration entre institutions muséales et milieux marchands nous serviront à comprendre les objectifs et les intérêts des acteurs 
concernés, mais aussi les conséquences sur l'institution muséale et sur notre manière d'appréhender l'objet ethnographique aujourd'hui.

Mots clé : objet, marché, musées d'ethnographie

\section{Abstract}

The ethnographical object between the market and heritage: a map and what is at stake

In recent years, ethnographic museums have been subject to ever closer scrutiny. When their collections are examined, so too is our approach to the contents: what cultural, affective, monetary, or scientific import do we give them and why? Academic research has already underlined several of these values and the complex network in which they come into being (collectors, museums, art dealers, audiences, etc). This article will focus on the relationship between two particular actors of this network (ethnographic museums and dealers in non-Western art). Two examples of collaborations between museums and dealers will shed light on the objectives and interests of the actors, but also on how they affect museums as a whole, and how they change our current approach to ethnographical objects.

Key words: object, market, ethnographic museums 